\title{
La influencia de dialectos chinos en el aprendizaje de la pronunciación de español ${ }^{1}$
}

\author{
CHEN DANNA \\ Universidad de Estudios Internacionales de Shanghái / Instituto Confucio de Madrid \\ danna.chen@shisu.edu.cn \\ WANG LILI \\ Universidad de Las Palmas de Gran Canaria \\ lili.wang@ulpgc.es
}

\begin{abstract}
Resumen: Este trabajo pretende mostrar la influencia fonológica de los dialectos chinos en el aprendizaje de español tomando el shanghainés como ejemplo. Se hace una comparación entre el inventario consonántico y vocálico, así como la entonación del dialecto shanghainés y del chino mandarín para encontrar las características fonológicas propias del primero que ayudan en el aprendizaje del español. La investigación revela que se facilita la pronunciación de las consonantes oclusivas sonoras y la entonación menos marcada para los hablantes del shanghainés gracias a su dialecto, pero no les resultan fáciles los grupos consonánticos ni la vibrante. El gran número de consonantes nasales en el chino mandarín dificulta la distinción entre las sílabas que contienen los sonidos nasales y aquellas sin ellos. Por último, se propone el trabajo colaborativo entre el profesorado nativo y no nativo para emprender una enseñanza fonética correcta y eficaz.
\end{abstract}

Palabras clave: estudio contrastivo, pronunciación de español, dialecto de China, shanghainés

\section{The influence of Chinese dialects in learning Spanish pronunciation}

Abstract: This paper aims to show the phonological influence of Chinese dialects in Spanish learning Spanish taking Shanghainese as an example. A comparison between the consonantal and vocalic inventory, as well as the intonation of the Shanghai dialect (Shanghainese) and Mandarin Chinese are made to find the phonological characteristics of the Shanghai dialect that contribute to the learning of Spanish. The research reveals that the Shanghai dialect facilitate the learning of the pronunciation of occlusive voiced consonants and the intonation in Spanish, however, like other Mandarin Chinese speakers, the Shanghainese speakers also find it difficult to pronounce the consonant groups and the alveolar trill. The large number of nasal consonants in Mandarin Chinese makes it hard to distinguish between the syllables that contain the nasal sounds and those without them. Finally, the collaborative work between native and non-native teachers is proposed to undertake a correct and effective phonetic teaching.

Keywords: contrastive study, Spanish pronunciation, Chinese dialect, Shanghainese

\footnotetext{
${ }^{1}$ Este trabajo está enmarcado en el proyecto KY01X0222017064 financiado por la Universidad de Estudios Internacionales de Shanghái (本论文获中央高校基本科研业务费资助)。
} 
El sistema fonológico de una lengua ya aprendida influye de forma positiva o negativa en el aprendizaje de la pronunciación de la nueva. Si es el idioma materno (o el primer idioma) del aprendiente, la transferencia puede ser fuerte y duradera. Existen estudiosos que se han dado cuenta de las dificultades de hablantes del chino mandarín (Pǔtōnghuà 普通话) en el aprendizaje de español causadas por la falta de algunos elementos fonéticos en el idioma asiático. Sin embargo, en poca investigación se percibe la atención a la influencia de los dialectos que se hablan en China. En realidad, en el país asiático hay numerosos dialectos que son lenguas de familia y se aprenden antes del chino mandarín, por lo que ejercen una influencia notable sobre sus hablantes en el aprendizaje de la pronunciación de los idiomas extranjeros, en nuestro caso, el español. De modo que cuando hablamos de la enseñanza de la fonología de español a sinohablantes, debemos tomar muy en cuenta la existencia de los dialectos en China.

\section{Estudios antecedentes y descubiertos nuevos}

Constituyen los enfoques principales de los primeros estudios sobre las dificultades de sinohablantes en el aprendizaje de la pronunciación de español las oposiciones sonorasorda de las consonantes oclusivas $(/ \mathrm{p} /-/ \mathrm{b} /, / \mathrm{t} /-/ \mathrm{d} /, / \mathrm{k} /-/ \mathrm{g} /)$, la distinción entre las aspiradas y no aspiradas $\left(/ \mathrm{p} / \mathrm{y} / \mathrm{p}^{\mathrm{h}} /, / \mathrm{t} / \mathrm{y} / \mathrm{t}^{\mathrm{h}} /, / \mathrm{k} / \mathrm{y} / \mathrm{k}^{\mathrm{h}} /\right)$, la diferencia entre las vibrantes $/ \mathrm{r} /, / \mathrm{s} / \mathrm{y}$ la aproximante lateral alveolar /1/, la fricativa alveolar sorda /s/ y la dental sorda / $\theta /$ (Ortí Mateu 1990; Cao 2006, 2011; Cortés Moreno 2005, 2007, 2009; Planas Morales 2008; Chen 2011). Los análisis recientes encuentran más desafíos tales como la confusión entre la oclusiva bilabial sonora /b/ y la aproximante labiovelar sonora /w/ (Lu 2014: 7-16), el timbre inestable de la vocal /a/, la substitución de la vocal media anterior no redondeada /e/ por la media central /a/, y la realización como /u/ de la letra «o» (Igarreta Fernández 2015: 189-196).

En el plano suprasegmental, aunque Planas Morales (2008: 499) considera que los estudiantes chinos pueden tener muchas dificultades con el sistema acentual español y Ortí Mateu (1990: 7) atribuye dicho obstáculo al hecho de que el chino es idioma tonal y el español, entonacional el análisis de Cortés Moreno (2005: 1) revela que el aprendizaje de la acentuación no les plantea a sinohablantes dificultades notables y «las máximas dificultades se presentan en los contornos enfáticos». Por otra parte, Planas Morales (2009: 499) hace mención de la existencia de pausas inadecuadas en el enunciado articulado por sinohablantes, «que van desde pequeñas desconexiones entre elementos de un mismo grupo fónico a interrupciones bruscas en la velocidad de elocución».

Las conclusiones de los estudios anteriormente invocados formarán una lista larga de dificultades que encontrarían los sinohablantes al aprender la pronunciación de la lengua de Cervantes. Tras haber escuchado más de trescientas grabaciones de examinados chinos en el examen oficial de nivel de español en los centros universitarios chinos conocido como Examen de Español como Especialidad (EEE4), quisiéramos añadirle a dicha enumeración más escollos que se presentarían en el aprendizaje fonético ${ }^{2}: 1$ ) la confusión entre /un/ y /uən/, /an/ y /æn/; 2) la equivocación entre sílabas con la nasal y sin ella, por ejemplo: Confucio - *confuncio; avanzada - *avazada; 3 ) la dificultad en la distinción entre $/ \mathrm{n} / \mathrm{y} / 1 /$.

\footnotetext{
${ }^{2}$ Se usan las grabaciones de los años 2012 y 2013 como fuente de datos porque desde 2015 cancelaron la parte oral en EEE4.
} 
Las imperfecciones en la articulación como el primer problema no conducen a malentendidos, pero las dificultades 2) y 3) sí intervendrán la comunicación. El 1) y 2) pueden verse como las transferencias negativas del chino mandarín. Lu propone una explicación para el fenómeno número 1):

[...] en el chino estándar, cuando la nasal alveolar /n/ se encuentra al final de la sílaba, forma con la vocal una vocal nasal, en este caso la articulación de la vocal simple sufre una variación conforme a /n/ con la posición hacia adelante ( $\mathrm{Lu} 2014: 11$ ).

Completamos el planteamiento de Lu: la confusión de sinohablantes entre /un/ y /uən/ también se debe a la variación de /u/ delante de la nasal. Además, según lo estipulado en la transcripción fonética (pīnyīn 拼音) del chino mandarín, cuando «uen» se encuentra detrás de los iniciales (shēngmǔ 声母) se convierte en «un». Es decir, en la transcripción romanizada del chino mandarín, «un» es la abreviatura de «uen» en la escritura y se debe pronunciar como /uən/, por lo que se puede generar una transferencia negativa en el aprendizaje de español.

Pues bien, nos interesa conocer la causa del obstáculo número 3). Según Chen (2018: 221), los alumnos provenientes de Hunan, Hubei, Sichuan, Guangdong, etc. suelen tener este problema por «falta de la consonante nasal /n/ en sus dialectos». No es la única investigación que menciona la influencia de los dialectos chinos en la articulación de español por parte de sinohablantes. Cao (2011: 1-3) y Chen (2011: 55-60) revelan que los alumnos que hablan algún dialecto $\mathrm{Wu}$ cuentan con más facilidad en el aprendizaje de la pronunciación de español. De acuerdo con la investigación de Chen (2011: 55), la mayoría de los alumnos que cuentan con una pronunciación relativamente buena en español proviene de la ciudad de Shanghai (14 entre 15), mientras que 8 entre los 15 que poseen un nivel bajo al respecto son del norte de China, lo cual constituye la motivación del presente trabajo, en el que se pretende encontrar los elementos fonológicos propios de algunos dialectos en China y su influencia en el aprendizaje de español.

\section{Los dialectos chinos y su influencia en el aprendizaje de español}

\subsection{Los dialectos chinos}

«China tiene 56 etnias. Es un país multiétnico, multilingüe, multidialectal y posee múltiples tipos lingüísticos. [...] Tenemos más de 130 lenguas y una treintena de escrituras» (Ministerio de Educación de China, 2015). Según el Ministerio de Educación de China, en el país asiático existen al menos siete grupos dialectales (Norte de China, Wu, Xiang, Gan, Kejia, Min y Yue), que en muchos casos no son comprensibles entre sí.

Habría que destacar la distinción entre el «dialecto» y los «grupos dialectales», puesto que en algunos estudios se usan los dos conceptos indistintamente con términos como «el dialecto Xiang» $\mathrm{o}$ «el dialecto $\mathrm{Wu}$ », etc. Tomando «Wu» como ejemplo, en realidad es el nombre de una familia de dialectos de más de 81 millones de hablantes (Ethnologue 2017: 1), en la que hay numerosos idiomas autóctonos de diferencias notables sobre todo en la pronunciación. Si no existieran caracteres chinos y se empleara una escritura alfabética para representar los sonidos de los dialectos de la familia $\mathrm{Wu}$, se inventarían incontables escrituras. Además, si se pregunta a un ciudadano de Shanghái si habla el dialecto $\mathrm{Wu}$, no sabrá contestarle porque el código lingüístico regional que utiliza se llama shanghainés y no suele decir "hablo el dialecto Wu". Encima, el shanghainés no es el único dialecto usado por los autóctonos. Se hablan en la zona periférica de la ciudad 
los dialectos de Songjiang, Jinshan, Fengxian, Chongming etc. que suenan bastante distintos.

Las distinciones principales entre los dialectos chinos consisten en la pronunciación, puesto que comparten buena parte de la gramática y el vocabulario (Yuan 2001:8), y se utiliza la misma escritura: los caracteres chinos. Es de conocimiento común que las variedades del chino en las zonas al norte del río Yangtsé disfrutan de una similitud mayor que aquellos del sur, donde se encuentra la mayoría de los grupos dialectales. Por otra parte, como la pronunciación del chino mandarín, lengua oficial de China, se basa en el dialecto pekinés, uno del norte, los alumnos de esa zona cuentan con una articulación relativamente unánime en comparación con los del sur, ya que el dialecto que habla suena parecido a la lengua oficial del país. De modo que las dificultades que se les presentan en la pronunciación de español también son similares y suelen ser las que se han citado en el apartado anterior. En cambio, el aprendizaje de la pronunciación de español supone otra cosa para alumnos del sur de China porque en su familia se habla un dialecto totalmente diferente que el chino mandarín en cuanto a la fonética. Por esta razón, hay que estudiar el sistema fonológico de los dialectos para conocer las verdaderas ventajas y desafíos de alumnos chinos en el aprendizaje de pronunciación.

\subsection{Comparación entre shanghainés, chino mandarín y español}

Los estudios contrastivos entre los sistemas fonológicos de las lenguas contribuyen a la prevención de errores en la pronunciación y la identificación de dificultades articulatorias. Hemos mencionado que los estudios de Cao (2011: 1-3) y Chen (2011: 54) revelan las ventajas de estudiantes de Shanghái en la articulación en español. En la gráfica 1 se presentan las diferencias cuantitativas entre las vocales y consonantes del chino mandarín, shanghainés y español.

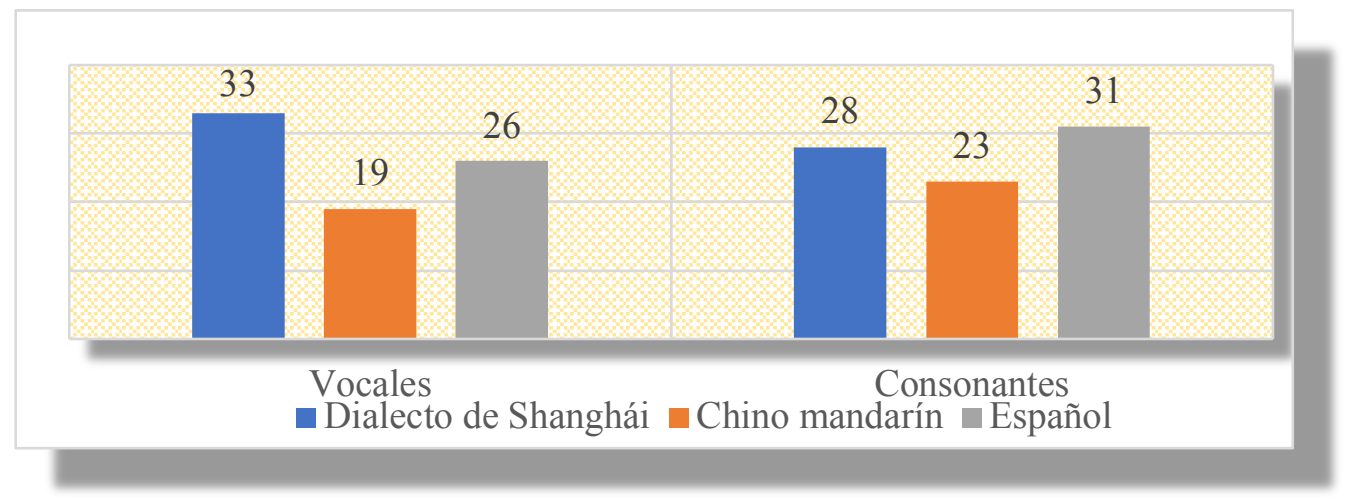

Gráfica 1. Elementos fonológicos en shanghainés, chino mandarín y español

Se computan los diptongos y triptongos en español y las combinaciones de vocales en chino mandarín y en shanghainés, pero no se incluyen las vocales que terminan con la consonante nasal, por ejemplo /uən/ en el primero e /yn/ en el segundo, aunque estas combinaciones se consideran «vocales» en la fonología del chino con el nombre de «finales» (yùnmǔ 韵母). Por otra parte, las consonantes, conocidas como «iniciales» (shēngmǔ 声母) en el sistema fonológico de la lengua china, comparten más similitudes con las en lenguas indoeuropeas.

Como existen diferentes entendimientos con respecto al sistema fonológico de los dialectos, usamos para este trabajo uno de los más reconocidos: Gran diccionario de 
shanghainés (Qian 2007: 386), según el cual dicha lengua vernácula tiene 41 vocales (finales) y 28 consonantes (iniciales). Entre las primeras hay 6 que terminan con la consonante nasal y $2(/ \mathrm{m} / \mathrm{y} / \dot{\mathrm{y}} /)$ que son consonantes silábicas, de modo que se calculan 33 vocales (y sus posibles combinaciones) según los criterios de la fonología del español. El chino mandarín cuenta con 19 vocales y combinaciones de vocales excluidas las de finales nasales (/an/ y / uy/ por ejemplo). La totalidad de vocales, diptongos y triptongos en español llega a 26. Se ve que el chino mandarín tiene menos variedades en cuanto al número de vocales. Pasa lo mismo con las consonantes: el shanghainés tiene 28, el chino mandarín, 23 y el español posee 31 consonantes y grupos consonánticos.

La comparación revela que el chino mandarín tiene menos elementos fonológicos entre los tres idioma-dialectos. Los estudiantes que hablan shanghainés aprenderán con más facilidad las vocales pero encontrarán dificultades en las consonantes, puesto que el español cuenta con mayor cantidad de estas. Las gráficas 2 y 3 muestran las diferencias detalladas entre los tres.

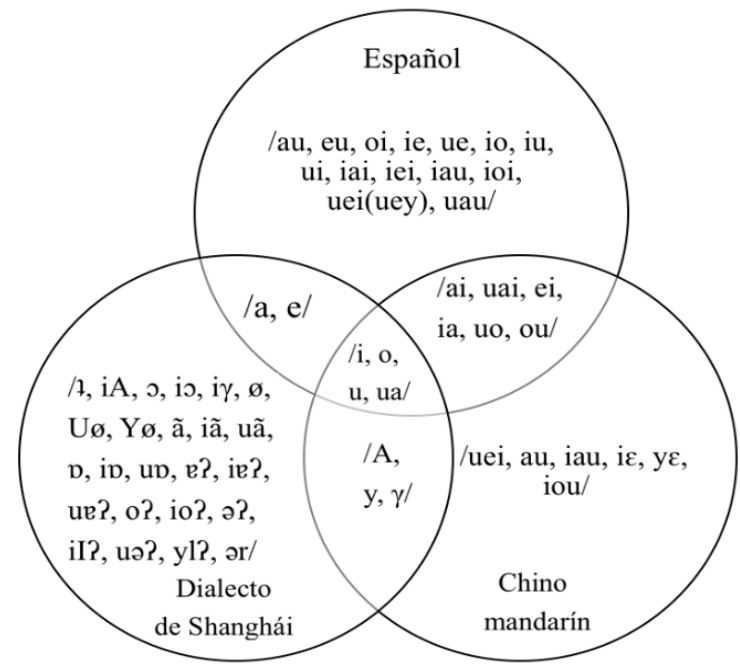

Gráfica 2. Vocales en shanghainés, chino mandarín y español

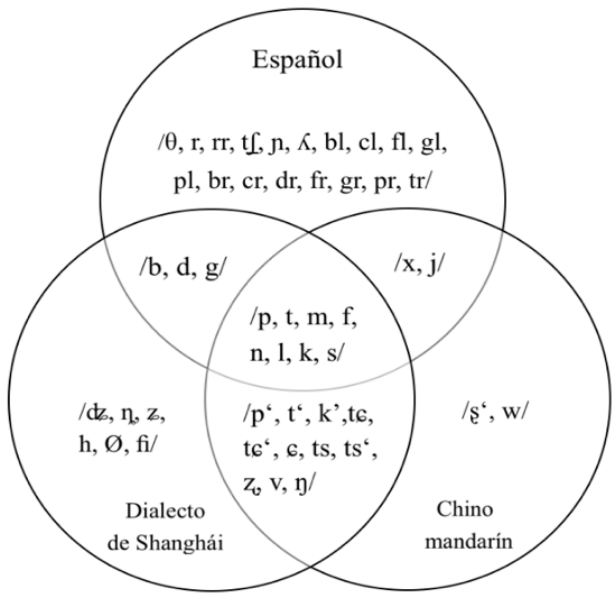

Gráfica 3. Consonantes en shanghainés, chino mandarín y español 
En cuanto a las vocales, el shanghainés y el español comparten los importantes /a/ y /e/, que solo aparecen en combinaciones vocálicas en chino mandarín. Además, llama la atención la existencia de la / / / en los dos códigos asiáticos, puesto que nos recuerda la substitución frecuente de la /e/ por la /a/ de sinohablantes. En las vocales semicerradas, /a/ se sitúa entre /e/ (en español y en shanghainés) y / / / (en chino). Se puede considerar un fonema "de tránsito" que articulan algunos hablantes de chino mandarín para llegar a /e/ pero no lo logran. En cambio, los hablantes de shanghainés sí saben pronunciarlo gracias al dialecto.

Con respecto a las consonantes, se ve con claridad en la Gráfica 3 que el shanghainés cuenta con las conocidas oclusivas $/ \mathrm{b} /, / \mathrm{d} / \mathrm{y} / \mathrm{g} /$ que suponen una dificultad enorme para los que solo hablan chino mandarín. De modo que no les cuesta a los hablantes de este dialecto aprenderlas. Por otro lado, también se notan los desafíos que comparten los hablantes de shanghainés y los monolingües de chino mandarín, tales como la /r/ y los grupos consonánticos en español.

Otra observación interesante es que más del $40 \%$ de las sílabas en chino mandarín finaliza con la nasal, de las cuales la mitad termina con la nasal velar $/ \mathrm{y} /$ mientras que en shanghainés las nasales velares se usan mucho menos, por lo que uno confundirá con más frecuencia las sílabas con nasales y las sin ellas si solo habla chino mandarín.

En el aspecto suprasegmental se percibe una ventaja evidente del dialecto de Shanghái también. Los cuatro tonos del chino mandarín se transcriben en la Gráfica 4 con los grados de 1 a 5 según la altura de entonación: el «55» se refiere al primer tono, el «35» al segundo tono, el «214» al tercer tono y el «51» al cuarto tono según las normas de la representación fonética. Aunque tanto el shanghainés como el chino mandarín poseen cinco grados de entonación, se demuestra en la Gráfica 4 que el segundo tiene una inclinación más radical de tonos que el primero. Comparado con el chino mandarín, el shanghainés presenta una entonación más suave. Además, en chino mandarín los caracteres tienen una pronunciación casi fija, solo algunas palabras cambian de tonos, por ejemplo, yī ( 一), bù (不), a (啊) y las palabras de dos sílabas que son de tercer tono ambas. Sin embargo, en shanghainés la entonación de los caracteres altera de acuerdo con la cantidad de sílabas (Gráficas 5 y 6). De modo que el shanghainés cuenta con una entonación más flexible y rica, lo que es favorable al aprendizaje de la pronunciación de una lengua entonacional como español.

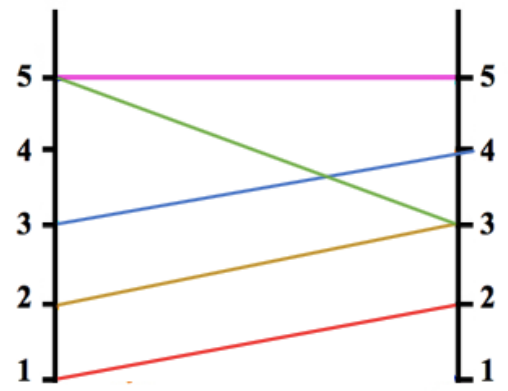

Shanghainés

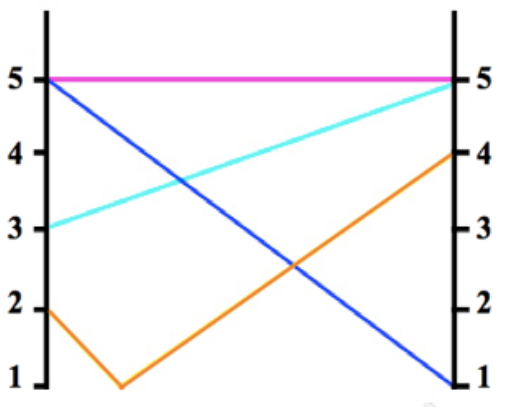

chino mandarín

Gráfica 4. Los tonos del dialecto de Shanghái y de chino mandarín 


\begin{tabular}{|c|c|c|c|c|}
\hline Una sílaba & Disílaba & Trisílaba & Cuatro sílabas & Cinco sílabas \\
\hline 53 & $55+21$ & $55+33+21$ & $55+33+33+21$ & $55+33+33+33+21$ \\
\hline 34 & $33+44$ & $33+55+21$ & $33+55+33+21$ & $33+55+33+33+21$ \\
\hline 23 & $22+44$ & $22+55+21$ & $22+55+33+21$ & $22+55+33+33+21$ \\
\hline 55 & $33+44$ & $33+55+21$ & $33+55+33+21$ & $33+55+33+33+21$ \\
\hline 12 & $11+23$ & $11+22+23$ & $22+55+33+21$ & $22+55+33+33+21$ \\
\hline
\end{tabular}

Gráfica 5. Cambios de tonos del dialecto de Shanghái (Qian 2007:387)

\begin{tabular}{|c|c|}
\hline Palabra & Cambios \\
\hline yī (一) & $\begin{array}{c}\text { yí+51 } \\
\text { yì +55/35/214 }\end{array}$ \\
\hline bù (不) & $\begin{array}{c}\text { bú+51 } \\
\text { bù+35/214 }\end{array}$ \\
\hline A (啊) & ā,á, ă, à \\
\hline $214+214$ & $35+214$ \\
\hline
\end{tabular}

Gráfica 6. Cambios de tonos de chino mandarín (Qian 2007:387)

Al cierre de este apartado, se presentan unos desafíos fonéticos de los hablantes de otros dialectos en China. Por ejemplo: algunos de Hunan, Anhui y Sichuan no distinguen entre /n/ y /1/. En el sur del país como Fujian, Hunan, Jiangxi, etc. no se nota la diferencia entre /f/ y /h/. Por otra parte, se plantean las ventajas de que disfrutan los alumnos de Taiwán y Xinjiang al aprender la pronunciación de la nasal palatal $/ \mathrm{y} / \mathrm{y}$ la vibrante múltiple $/ \mathrm{r} /$ gracias a los elementos fónicos similares en sus dialectos (Chen 2011, Muñoz Torres 2014), lo que demuestra la conciencia de los docentes sobre la influencia de los dialectos chinos en el aprendizaje de la pronunciación de español.

En realidad la situación es todavía más complicada porque en el país asiático, la mayoría de los aprendientes de español tiene el inglés como primera lengua extranjera y empiezan a estudiarlo desde una edad temprana. Dicha lengua masivamente estudiada generará transferencias tanto positivas como negativas en el aprendizaje de español. Es decir, antes de aprender la pronunciación del idioma de Cervantes, gran parte de estudiantes sinohablantes tienen ya conocimientos de tres sistemas fonéticos: el de chino mandarín, el de su dialecto y el de inglés, cuyos elementos fónicos afectarán a su aprendizaje de la 
nueva lengua. Como los primeros son lenguas de educación y de familia, ejercen una influencia mayor que la última.

\section{Sugerencias didácticas}

Tanto docentes españoles como chinos han planteado sugerencias prácticas para facilitar y mejorar la pronunciación de los alumnos sinohablantes. Se recomienda el estado relajado durante la articulación (Chen 2011: 38) y se diseñan ejercicios de identificación de elementos fónicos (Cortés Moreno 2007, Muñoz Torres 2014). Como señala Lu (2014:15), «hay que hacer a sinohablantes explicaciones y darles muchos ejercicios de identificación e imitación de sonidos desde la etapa inicial, ejercicios intensivos y estructurales, muchas veces repetitivos e incluso monótonos». En resumen, "atención, explicación, imitación y repetición" son cuatro pasos cruciales. Hay que ser consciente de las posibles dificultades que se producirán durante el aprendizaje de la pronunciación debido a la falta de elementos fonéticos en su lengua materna; se debe explicar el concepto de los fonemas desconocidos y la forma de articularlos; es importante hacer al alumnado imitar los sonidos difíciles de pronunciar y por último, una vez aprendida la pronunciación, se requieren algunos ejercicios repetitivos para perfeccionarla.

Estamos de acuerdo con los planteamientos anteriormente mencionados y quisiéramos destacar la necesidad de la cooperación entre el profesorado nativo y el local en este proceso si se estudia la lengua en China, donde la mayoría del profesorado es nativo del chino mandarín y el dialecto regional. Naturalmente los profesores no nativos se han enfrentado y seguirían enfrentándose con las dificultades en la pronunciación de español a causa de las transferencias negativas de su lengua materna y transmitirían dichos desafíos a sus alumnos. En este caso, son de enorme importancia la atención del profesorado local a las deficiencias en su propia producción fonética y la autocorrección. La enseñanza de la pronunciación se encuentra en las primeras fases de la docencia de la lengua extranjera, de modo que en China suelen impartir las clases de fonética los profesores locales, quienes son los que explican y articulan como modelo para sus alumnos. Dicha realidad afectará de forma negativa a la pronunciación de los aprendientes si los profesores se ven influidos también por los acentos dialectales y de chino mandarín. En este sentido se recomendaría realizar un trabajo colaborativo (co-enseñanza) entre el profesorado local y el nativo: el primero se encargará de la "atención" y la "explicación" de los cuatro pasos citados en el texto anterior, mientras el segundo servirá del objeto de "imitación" y diseñará ejercicios de "repetición". En otras palabras, el profesor local indica las posibles dificultades y explican la forma de articulación. Al mismo tiempo, el nativo muestra el sonido para que los alumnos lo imiten y prepara actividades de refuerzo. Además, es aconsejable que los dos profesores realicen investigaciones similares al presente trabajo para que ambos conozcan las características fonéticas de la lengua materna de sus alumnos.

\section{Conclusión}

Influido por el sistema fonológico de los dialectos, los sinohablantes se enfrentan con dificultades articulatorias de distintos niveles en el aprendizaje de la pronunciación de español. Es recomendable que el docente conozca el origen del alumnado y las características fonéticas de los dialectos de su región. Los estudios contrastivos como el que se ha realizado en este trabajo contribuirán al conocimiento sobre los códigos lingüísticos regionales en China y las posibles facilidades y/o dificultades que tendrá el alumnado en su aprendizaje. Como buena parte del profesorado de español en China no 
es nativo, se verá influido también por el sistema fonológico de su lengua materna. De allí surge la necesidad de la colaboración entre los profesores chinos y los nativos. Los primeros llaman la atención al alumnado a los desafíos con los que se encontrarán, explican el concepto y la forma de articulación. Los segundos proporcionan las pronunciaciones modelo y diseñan ejercicios para que practiquen los alumnos dentro y fuera de clase.

\section{Bibliografía}

CAO, Yufei (2006). «Un estudio contrastivo de los fonemas oclusivos entre español y chino: reflexiones de la enseñanza de la fonética española a estudiantes chinos», Sincronia, 2. Recuperado el 27/4/19, de: http://sincronia.cucsh.udg.mx/caoyufei06.htm

CAO, Yufei (2011). «La pronunciación del español para sinohablantes: una perspectiva dialectal», Congreso Mundial de Profesores de español. Recuperado el 24/3/19, de: http://comprofes.es/sites/default/files/slides/cao_yufei_elisa.pdf

CHEN, Xing (2018). «Análisis de los errores pragmáticos en el nivel fónico cometidos por los alumnos sinohablantes», Monográficos SinoELE, 17, 217-225.

CHEN, Zhi (2011). «Errores articulatorios de los estudiantes chinos en la pronunciación de las consonantes españolas», Monográficos SinoELE, 4, 54-67.

CORTÉS MORENO, Maximiano (2005). «Análisis experimental del aprendizaje de la acentuación y la entonación españolas por parte de hablantes nativos de chino», Phonica, $1,1-25$.

CORTÉS MORENO, Maximiano (2007). «Enseñanza de la pronunciación a alumnos chinos», DidactiRed. Recuperado el 24/3/19, de: https://cvc.cervantes.es/aula/didactired/anteriores/julio_07/16072007.htm

CORTÉs Moreno, Maximiano (2009). «De la fonología a la enseñanza de la pronunciación mediante juegos», MarcoELE, 8, 1-18.

EthNOLOGUE (2017). Chinese, $W u$. Recuperado 30/3/19, de https://www.ethnologue.com/language/wuu.

IGARRETA FERNÁNDEZ, Alba (2015). «La corrección de la pronunciación de los estudiantes sinohablantes en el aula de E/LE», Foro de profesores de E/LE, 11, 189-196.

LU, Jingsheng (2014). «Dificultades del sistema consonántico para sinohablantes desde el enfoque contrastivo», Monográficos SinoELE, 10, 7-16.

MiNISTERIO DE EDUCACIÓN DE CHINA (2015). 中国语言文字概况. Recuperado 30/3/19, de: http://www.moe.gov.cn/jyb_sjzl/s5990/201506/t20150610_189893.htm

MuÑOZ TORRES, Marta. (2014). «Dificultades fonético-fonológicas de los estudiantes chinos en el aprendizaje del español», Monográficos SinoELE, 10, 208-227.

OrTí MATEu, Rosa (1990). Comparación fonética, diagnóstico y tratamiento de las dificultades de los estudiantes chinos para aprender español. (Tesis Doctoral), Universidad de Filipinas, Dilimán, Quezon City.

Planas Morales, Silvia (2008). «Enseñanza de la pronunciación del español en estudiantes chinos: la importancia de las destrezas y los contenidos prosódicos». En La 
evaluación en el aprendizaje y la enseñanza del español como lengua extranjera/segunda lengua: XVIII Congreso Internacional de la Asociación para la Enseñanza del Español como lengua Extranjera (ASELE) (pp. 497-503), Alicante.

Planas Morales, Silvia (2009). «Enseñanza y evaluación de la pronunciación de E/LE en alumnos chinos», RedELE, 17. Recuperado el 24/3/19, de: http://www.educacionyfp. gob.es/dam/jcr:df5c6459-1273-436b-966a-5e749d27e570/2009-redele-17-04planaspdf.pdf.

钱乃荣 QIAN, Nairong. (2007). 上海话大词典. Gran diccionario de shanghainés. 上海 : 上海辞书出版社. Shanghai: Shanghai Cishu Publishing House.

袁家骅. YUAN, Jiahua (2001). 汉语方言概要. Introducción al dialecto de Shanghái. 北 京: 语文出版社. Beijing: Language \& Culture Press. 